\title{
EXTENDING FAMILIES OF DISJOINT ZERO SETS
}

\author{
C. E. AULL
}

\begin{abstract}
The $z$-cellularity $z(X)$ of a space $X$ is defined as $z(X)=\sup \{|Z|: Z \subset Z(X)\}$

where $Z(X)$ is the family of zero sets of $X$. It is proved using $\mathrm{CH}$ that a Tychonoff space $S$ is $T C^{*}$-embedded in every Tychonoff space it is $C$-embedded in iff $z(S) \leq c$. A space $S$ is defined to be $T C^{*}$-embedded in a space $X$ if any disjoint family of zero sets of $S$ can be extended to a family of disjoint zero sets of $X$. Similar theorems are proved for $C^{*}$-embedding when $S$ is a $P$-space or the zero sets have the Isiwata property.
\end{abstract}

1. Introduction. If a set $S$ is dense and $C$-embedded in a Tychonoff space $X$ the closure of a zero set of $S$ in $X$ is a zero set of $X$ [6]. Hence any family of disjoint zero sets of $S$ may be extended to a family of disjoint zero sets of $X$. A set $A$ of $S$ is said to be extended to a set $E(A)$ of $X$ if $E(A) \cap S=A$.

In this paper, we investigate the disjoint extension of families of disjoint zero sets of a set to a space, some families with restricted cardinals and some with restrictions to regular zero sets. Unless noted, definitions and terminology will be found in [6].

Theorem 1. A space $S$ is $C^{*}$-embedded in a space $X$ iff any denumerable family of disjoint zero sets may be extended disjointly to a family of zero sets of $X$.

Proof. Let $\left\{Z_{n}\right\}$ be a disjoint denumerable family of zero sets of $X$. Let $H_{n k}$ be a zero set extension of $Z_{n}$ to $X$ disjoint from $H_{k n}, k \neq n$, guaranteed by the $C^{*}$-embedding of $S$ in $X$. Then the family $\left\{H_{n}: H_{n}=\bigcap\left\{H_{n k}: k \neq n\right\}\right\}$ is the desired family of disjoint extensions of $\left\{Z_{n}\right\}$.

Definition 1. A set $S$ is $T C^{*}$-embedded $\left(T_{z}\right.$-embedded) in a space $X$ if any disjoint family of zero (cozero) sets of $S$ can be extended to a disjoint family of zero (cozero) sets of $X$.

In [1], $T_{z}$-embeddings were studied.

It is clear that any $C^{*}$-embedded zero set is $T C^{*}$-embedded and if every subset of a space is $T C^{*}$-embedded, then the space is perfectly normal and extremally disconnected.

2. $C$-embeddings and $T C^{*}$-embeddings. We can improve Theorem 1 with respect to $T C^{*}$-embeddings when the embedded set is $C$-embedded and dense as mentioned earlier. More generally we have the following theorem.

Theorem 2. Let $S$ be $C$-embedded in a Tychonoff space $X$. Then $\omega_{1}$ disjoint zero sets in $S$ may be extended to disjoint zero sets of $X$.

Received by the editors August 9, 1982 and, in revised form, February 7, 1983.

1980 Mathematics Subject Classification. Primary 54C50, 54C45; Secondary 54D60, 54G05, 54G10, 54G20. 
Proof. We order the $\omega_{1}$ zero sets $\left\{Z_{\alpha}\right\}$ by putting them into one-to-one correspondence with the countable ordinals. Let $E\left(Z_{\alpha}\right)$ be a zero set extension of $Z_{\alpha}$ for $\alpha<\omega_{1}$. By means of Lemma 2, we construct a zero set $Z_{\alpha}^{\beta}$ of $X$ such that $Z_{\alpha} \subset Z_{\alpha}^{\beta}$ and $Z_{\alpha}^{\beta} \cap E\left(Z_{\beta}\right)=\varnothing$ for each $\beta<\alpha$. The family $\left\{H_{\alpha}\right\}, H_{\alpha}=$ $\bigcap_{\beta}\left\{Z_{\alpha}^{\beta}: \beta<\alpha\right\} \cap E\left(Z_{\alpha}\right)$ is a family of disjoint zero set extensions of $\left\{Z_{\alpha}\right\}$.

Lemma 2. A $z$-embedded set $S$ is $C$-embedded in a space $X$ iff for $H$ a zero set of $S$ and $Z$ a zero set of $X, H \cap Z=\varnothing, H$ and $Z$ are completely separated in $X$.

Proof. Sufficiency. Since $S$ is $z$-embedded and completely separated from any disjoint zero set of $X, S$ is $C$-embedded in $X[3]$.

Necessity. Let $f \in C(X)$ and $h \in C(S)$ such that $Z=f^{-1}(0)$ and $H=h^{-1}(0)$. Then $|f|+|h|$ is continuous on $S$ and can be extended to a positive continuous function $g$ on $X$. Set $h^{\prime}=g-|f|$. Then $h^{\prime}=|h|$ on $S$. Since $g>0, h^{\prime}>0$ on $Z$. So $h^{\prime-1}(0)$ and $Z$ are disjoint zero sets of $X$ containing $H$ and $Z$ respectively.

REMARK 1 . It ic clear from the above proof that $C$-embedding of a set $S \subset$ $X$ is equivalent to $C^{*}$-embedding and every positive $f \in C^{*}(X)$ having a positive continuous extension. This is probably known.

Definition 2. ( $z$-cellularity) $z(X)=\sup \{|Z|: Z \subset Z(X), Z$ a disjoint family $\}$ where $Z(X)$ is the family of all zero sets of $X$.

Corollary 2. If $X$ is almost compact [6] with $z(X)=\omega_{1}$, then $X$ is $T C^{*}$ embedded in every Tychonoff space $X$ is embedded in.

The necessity of the above conditions follows from the next theorem.

THEOREM $3(\mathrm{CH})$. A Tychonoff space $X$ is $T C^{*}$-embedded in every Tychonoff space it is $C$-embedded $\left(C^{*}\right.$-embedded) in iff (only if) $z(X) \leq c$.

Proof. Sufficiency of the $C$-embedding result follows from Theorem 2 . Necessity is obtained by embedding $\nu X$ in the product of real lines, in which $X$ will be $C$ embedded in and by Engelking's [4, p. 295] result that $z(X) \leq c$ for the product of regular separable spaces. Thus if $z(X)>c$, there is a Tychonoff space in which $X$ is $C$-embedded in but not $T C^{*}$-embedded in. The $C^{*}$-embedding result follows.

REMARK 2 . We cannot replace $C$-embedding by $C^{*}$-embedding in the sufficiency argument as Ralph Fox has shown that $R$ is not $T C^{*}$-embedded in $\beta R$.

Corollary $3(\mathrm{CH})$. A Tychonoff space $X$ is TC $C^{*}$-embedded in every Tychonoff space it is embedded in iff $X$ is almost compact and $z(X) \leq c$.

\section{2. $C^{*}$-embeddings and $T C^{*}$-embeddings.}

THEOREM 4. If a family of $\omega_{1}$ disjoint zero sets of a Tychonoff space $S$ may be extended disjointly to $\beta S$, then this family of zero sets of $S$ may be disjointly extended to any Tychonoff space $S$ is $C^{*}$-embedded in.

Proof. If $S$ is $C^{*}$-embedded in $X, \beta S \subset \beta X$ and $\beta S$ is $C$-embedded in $\beta X$. By Theorem $2 \omega_{1}$ disjoint zero sets of $\beta S$ may be extended to $\beta X$. The result follows.

Corollary $4(\mathrm{CH})$. Let $S$ be Tychonoff and TC*-embedded in $\beta S$. Then $S$ is $T C^{*}$-embedded in every space it is $C^{*}$-embedded in iff $z(S) \leq c$. 
It is not unusual to have a set $S T C^{*}$-embedded in $\beta S$ even when there are families of disjoint zero sets of $S$ of large cardinality. This is always true when $S$ is pseudocompact or discrete. In these cases, by Theorem 4 , any family of $\omega_{1}$ disjoint zero sets may be extended disjointly to a family of zero sets of any Tychonoff space in which $S$ is $C^{*}$-embedded in. The next two theorems are modifications of these results.

THEOREM 5. Let $\left\{z_{\alpha}\right\}$ be a family of disjoint zero sets of cardinality $\omega_{1}$ of a set $S$ such that for $f \in C^{*}(S)$ such that $Z(f) \cap Z_{\alpha}=\varnothing,|f(z)| \geq \epsilon>0$ for $z \in Z_{\alpha}$. Then $\left\{Z_{\alpha}\right\}$ may be extended disjointly to any space in which $S$ is $C^{*}$-embedded.

Proof. Let $E\left(Z_{\alpha}\right)$ be a zero set extension of $Z_{\alpha}$ to $X$. Thus as in Lemma 2, $E\left(Z_{\alpha}\right)$ is completely separated in $X$ from $Z_{\beta}$ for $\beta \neq \alpha$. The proof is completed as in Theorem 2.

The property referred to at the end of the first sentence of the statement of the theorem was discovered by Isiwata [7] to be satisfied by all zero sets of a pseudocompact space. We will refer to the above property as the Isiwata property. By modifying the proof of Theorem 5, one may prove the following result.

TheOREM 5A. let $S \subset X, X$ Tychonoff, have at most $\omega_{1}$ disjoint zero sets and let every disjoint family of zero sets, none of which has the Isiwata property be denumerable. Then $S$ is $T C^{*}$-embedded in $X$ if $S$ is $C^{*}$-embedded in $X$.

Definition 3 (COMPARE [2]). A weak $0 z$-space is a space where the closure of a cozero set is a zero set.

We will call a zero set that is also a regular closed set a regular zero set.

THEOREM 6. Let $\beta S$ be weak $0_{z}$. If $\left\{Z_{\alpha}\right\}$ is a disjoint family of regular zero sets (of cardinality $\omega_{1}$ ) then $\left\{Z_{\alpha}\right\}$ may be extended disjointly to a family of zero sets of $\beta S$ (of $X$ Tychonoff where $S$ is $C^{*}$-embedded in $X$ ).

Proof. Since $\beta S$ is weak $0_{z}, S$ is weak $0_{z}$. So the interior of $Z_{\alpha}$ is a cozero set. From [1], \{int $\left.Z_{\alpha}\right\}$ has a family of disjoint cozero extensions $\left\{C_{\alpha}\right\}$ in $\beta S$. It is immediate that in $\beta S, \bar{C}_{\alpha}=\bar{Z}_{\alpha}$ so that $\bar{Z}_{\alpha}$ is a zero set of $\beta S$. By the $C^{*}$ embedding of $S$ in $\beta S$, these zero sets are necessarily disjoint. An application of Theorem 4 completes the proof.

An important class of spaces that satisfy the conditions of the theorem are basically disconnected spaces.

Corollary 6. Let $S$ be a $P$-space. Then $S$ is $T C^{*}$-embedded in $\beta S$ and any family of $\omega_{1}$ disjoint zero sets of $S$ may be extended disjointly to any Tychonoff space $S$ is $C^{*}$-embedded in.

Proof. Since $S$ is a $P$-space, $\beta S$ is basically disconnected and each zero set of $S$ is a regular closed set.

4. Some examples. The author is indebted to E. van Douwen for pointing out the following theorem of Juhasz which will be useful in analyzing examples.

TheOREM A (Juhasz [8]). If $X$ is compact (even if $X$ is the union of $G_{\delta}$-sets of some compactification, i.e. $X$ is of point-countable type) and $S$ has cellularity $m, m$ infinite, then $X$ has at most $2^{m}$ disjoint $G_{\delta}$-sets. 
In the above theorem, we can replace $G_{\delta}$-sets by zero sets and compact by pseudocompact sets based on Theorem 2 .

We have already noted $\mathrm{R}$. Fox's result that $R$ is not $T C^{*}$-embedded in $\beta R$. However as all disjoint families of regular zero sets are countable, these families are disjointly extendable. By Theorem 5, using $\mathrm{CH}$, all disjoint families of compact zero sets of $R$ are disjointly extendable.

The following example of R. Pol, pointed out to the author by R. Hodel, shows that even in the case of dense $C^{*}$-embedding we may be limited in the cardinality of the number of disjoint zero sets that are extended in case $S$ is not pseudocompact or a $P$-space.

EXAMPLE 1 (R. Pol [9]). The space $S$ is Tychonoff, satisfies c.c.c., has countable pseudocharacter and has cardinality $2^{c}$. Thus $S$ has $2^{c}$ disjoint zero sets. Furthermore $\beta S$ satisfies c.c.c; so by Theorem A the $z$-cellularity of $\beta S$ is a most c. So $S$ is not $T C^{*}$-embedded in $\beta S$.

ExAmple 2. Let $X$ be the absolute of $S$; then $\beta X$ is the absolute of $\beta S[\mathbf{1 1}]$ and since c.c.c. is preserved under taking absolutes $[11], X$ satisfies c.c.c. and since there is a continuous map from $X$ to $S, z(x) \geq 2^{c}$. By Theorem $\mathrm{A}$ and the fact that $\beta X$ satisfies c.c.c., the $z$-cellularity of $\beta X$ is at most $c$. Thus $X$ is not $T C^{*}$-embedded in $\beta X$ and $X$ is extremally disconnected.

ExAmPLE 3. Since $\beta N \sim N$ is a zero set of $\beta N, \beta N \sim N$ is $T C^{*}$-embedded in $\beta N$ and is not $T_{z}$-embedded in $\beta N$. Even though $C^{*}$-embedding implies $z$ embedding $T C^{*}$-embedding does not imply $T_{z}$-embedding.

Blair [2] has shown that if a weakly perfectly normal space is not realcompact one can add a point of $\nu X \sim X$ and obtain a weakly perfectly normal space that is not perfectly normal (the additional point is not a $G_{\delta}$ in the space). We will call this space $W(X)$. It is clear that if $X$ is normal and hereditarily extremally disconnected then so will $W(X)$ be.

EXAMPLE 4 (BLAIR [2]). If $D$ is a discrete space of measurable cardinality $W(D)$ is a hereditarily extremally disconnected normal space that is not perfectly normal. Furthermore it is easily shown that every subset of $W(D)$ is $T C^{*}$-embedded so that every subset of a space may be $T C^{*}$-embedded without the space being perfectly normal.

Below we have another example of this where we use the set-theoretic axiom club.

EXAMPLE 5 (WAGE [10]). The space $X$ is perfectly normal and extremally disconnected and constructed using club. Then $W(X)$ is normal and hereditarily extremally disconnected and is not perfectly normal.

The question then arises as to whether normal hereditarily extremally disconnected spaces have the property that every subset is $T C^{*}$-embedded, particularly under $\mathrm{MA}+\sim \mathrm{CH}$. tions.

Finally, the author would like to thank the referee for his many helpful sugges-

\section{REFERENCES}

1. C. E. Aull, Extendability and expandability (submitted for publication).

2. R. L. Blair, Spaces in which special sets are z-embedded, Canad. J. Math. 28 (1976), 673-690.

3. R. L. Blair and A. W. Hager, Extensions of zero-sets and of real-valued functions, Math. Z. 136 (1974), 41-52. 
4. R. Engelking, Cartesian products and dyadic spaces, Fund. Math. 57 (1965), 287-306.

5. __ General topology, PWN, Warsaw, 1977.

6. L. Gillman and M. Jerison, Rings of continuous functions, Van Nostrand, Princeton, N. J., 1966.

7. T. Isiwata, Mappings and spaces, Pacific J. Math. 20 (1967),455-480.

8. I. Juhasz, Cardinal functions in topology, Math. Centre Tracts, No. 34, Mathematisch Centrum, Amsterdam, 1971.

9. R. Pol, Short proofs of two theorems of cardinality of topological spaces, Bull. Acad. Polon. Sci. Sér. Math. 22 (1974), 1245-1249.

10. M. Wage, Extremally disconnected $S$-spaces, Topology Proc. (Conf. Auburn Univ. Auburn, Ala, 1976), Math. Dept., Auburn Univ., Auburn, 1977, pp. 181-186.

11. R. G. Woods, Absolutes of topological spaces, Math. Centre Tracts, No. 116, Mathematisch Centrum, Amsterdam, 1979, pp. 323-362.

Department of Mathematics, Virginia Polytechnic Institute and State UNIVERSITY, BLACKSBURG, Virginia 24061 